\title{
PSICOLOGÍA POSITIVA: ANÁLISIS DESDE SU SURGIMIENTO
}

\section{POSITIVE PSYCHOLOGY: ANALYZING ITS BEGINNINGS}

\author{
María Laura Lupano Perugini \\ Universidad de Palermo, Argentina \\ Alejandro Castro Solano \\ Universidad de Palermo, Argentina
}

\begin{abstract}
Resumen: A más de diez años de la fundación de la Psicología Positiva por Martin Seligman, este trabajo se propone hacer un análisis desde sus inicios, considerando sus antecedentes, sus temas de interés, su producción, su difusión, sus implicaciones prácticas (intervenciones) y sus líneas de estudio a futuro. Además se propone discutir acerca del futuro de la Psicología Positiva: su disolución o integración al resto de la disciplina psicológica.
\end{abstract}

Palabras clave: Psicología Positiva, inicio, producción, intervenciones, futuro.

\begin{abstract}
More than a decade since the foundation of Positive Psychology by Martin Seligman, this paper aims to analyze this branch of psychology starting at its very beginning, considering its precedents, its topics of interest, its production, its diffusion, its implications for clinical practice, and future research lines. Furthermore, this paper introduces a discussion regarding the future of Positive Psychology: either its dissolution, or its integration with the other fields of psychology.
\end{abstract}

Keywords: Positive Psychology, beginning, production, interventions, future.

\section{PSICOLOGÍA POSITIVA: ANÁLISIS DESDE SU SURGIMIENTO}

La Psicología Positiva (PP) ya cuenta con más de diez años desde su fundación. Resulta relevante realizar un análisis desde sus inicios, considerando sus antecedentes, su temas de interés, su producción, sus implicaciones prácticas y sus líneas de estudio a futuro; pero aún más relevante resulta discutir cual será su futuro. En los siguientes apartados se abordan en profundidad dichos temáticas.

\section{Los inicios}

Existe consenso en considerar que el inicio de la Psicología Positiva se señala en 1998 con el discurso inaugural de Martin Seligman como presidente de la American Psychological Association (APA) (Seligman, 1999).

Seligman, tras haber tenido un encuentro el año anterior -1997- con su colega Csikszentmihalyi -en un centro vacacional- (Csikszent- mihalyi, 2003) y, un momento que el describe como epifánico con su hija Nikki (Seligman \& Csikszentmihalyi, 2000), se percata que la Psicología había estado abocada al estudio de la enfermedad en detrimento de los aspectos positivos.

Antes de la Segunda Guerra Mundial, la Psicología se había propuesto tres misiones: curar la enfermedad mental, ayudar a las personas a tener una vida más productiva y satisfecha, e identificar y promover el talento. Posterior a la Segunda Guerra, el acontecimiento de dos eventos cambiaron la orientación de la Psicología: en 1946 se creó la Veterans Administration y muchos psicólogos se abocaron al tratamiento de la enfermedad mental, y en 1947 se fundó el National Institute of Mental Health haciendo que muchos investigadores consideraran relevante el estudio sobre las diferentes psicopatologías. Los hechos mencionados hicieron que, de las tres misiones propuestas por la Psicología, solo se desarrolle la primera (curar la enfermedad mental).

Correspondencia: María Laura Lupano Perugini. Universidad de Palermo,Argentina. Correo Electrónico: mllupano@psi.uba.ar 
En parte, el surgimiento de la PP, es un intento de continuar con esas misiones olvidadas (Seligman \& Csikszentmihalyi, 2000). En el mencionado discurso inaugural, Seligman declara que su mandato tendrá como objetivo enfatizar el interés hacia una psicología más positiva: "La Psicología no es solo el estudio de la debilidad y el daño, es también el estudio de la fortaleza y la virtud. El tratamiento no es solo arreglar lo que está roto, es también alimentar lo mejor de nosotros" (Seligman, 2003, p.1).

En un primer momento, Seligman consideró que el énfasis debía estar en la prevención pero prontamente se dio cuenta que, de ese modo, seguía enfocado en la enfermedad, ya que el objetivo era meramente evitarla o minimizar sus efectos (Seligman, 2003).

La preocupación y el interés por el estudio del bienestar humano y los factores que contribuyen al mismo, no es exclusividad de la PP, sin embargo Seligman tuvo la virtud de identificar estas tendencias, nombrarlas y adaptarlas a los cánones ortodoxos de la ciencia, organizándola en una estructura programática e impulsando su investigación y difusión (Gancedo, 2008; Linley, Joseph, Harrington \& Wood, 2006).

\section{Los antecedentes}

Como se mencionó en el párrafo anterior, la preocupación por el bienestar y la felicidad no es un interés reciente. En cierto sentido, la Filosofía occidental no ha tenido nunca otra preocupación más central, bien desde el análisis directo de las condiciones sustantivas del bienestar (la eudamonía aristotélica) o bien, más modernamente, desde el análisis de las condiciones existenciales que limitan el alcance de ese ideal. Así que Aristóteles, pero también Spinoza, Schopenhauer, Bertrand Russell, Heidegger, o Cioran, han hecho de esta reflexión sobre la felicidad uno de los ejes del pensar sobre lo humano (Vázquez, 2006).

En el terreno de la Psicología, los antecedentes de la PP deben buscarse en los escritos de William James (James, 1902), de Allport (1958) y en los autores de la denominada Psicología Humanística, entre otras fuentes. Pueden citarse como referentes importantes los trabajos de autores como Rogers (1951, 1961), Maslow (1954, 1968); Jahoda (1958), Erikson (1963,
1982), Vaillant (1977); Deci y Ryan (1985); y Ryff y Singer (1996).

Además, si bien la historia de la PP es relativamente nueva, muchos psicólogos vienen haciendo PP sin saberlo. Los estudios clásicos sobre apego, inteligencia emocional, optimismo, conductas prosociales, valores humanos y creatividad, solo para nombrar algunos, son temas del campo de estudio de la PP (Castro Solano, 2009).

El mérito que corresponde a la PP es haber integrado en un corpus teórico pero con amplia validación empírica los mencionados tópicos de interés.

Para terminar de describir el inicio de la PP puede decirse que, tras el discurso inaugural de su fundador, se sucedieron una serie de encuentros en Akumal -México- entre investigadores interesados en el desarrollo de esta nueva corriente. Además se creó el Comité de Psicología Positiva y, posteriormente, el Centro de Psicología Positiva de la Universidad de Pensylvania, la primer Cumbre de Psicología Positiva en Washington DC y, se dedicó un número especial de la American Psychologist a los temas tratados por la incipiente PP. Todos estos hechos marcan definitivamente el inicio de este movimiento.

\section{Modelo Médico vs. Enfoque Salugénico}

El cambio de enfoque desde el centramiento en la enfermedad al desarrollo de las potencialidades, que Seligman percibe claramente en la búsqueda de su misión presidencial, podría denominarse enfoque salugénico que empieza a hacerse visible y completar al modelo médico hegemónico en la ciencia psicológica (Gancedo, 2008).

Gancedo (2008) describe el recorrido que va desde un enfoque al otro. El modelo médico se organizó desde y hacia la enfermedad (Ver RAE, 2001; Diccionario Terminológico de ciencias médicas, 1976). Diagnóstico, pronóstico, curación y prevención son términos de esta óptica. Este modelo luego se extendió a las Ciencias Sociales y comienza a manifestarse como insuficiente a mediados del siglo $\mathrm{XX}$ cuando la OMS, en 1947, destaca que la salud no es simplemente la ausencia de enfermedad. 
En la década del 80 del siglo pasado, germina el concepto de promoción de la salud que anticipa el surgimiento del enfoque salugénico. Desde la promoción no se busca solo la evitación (prevención) de la enfermedad sino fundamentalmente el incremento de la salud y el bienestar en general (Godoy, 1999).

Antonovsky $(1979,1987)$ desde el ámbito de la Sociología de la Medicina, postula la Orientación Salugénica viendo a la salud/ enfermedad como extremos de un continuo mas que como posiciones dicotómicas y se interesa por estudiar aquellos factores que promueven movimientos hacia el extremo favorable del continuo. En el terreno de la Psicología, la primera escuela en advertir las limitaciones del modelo médico fue la Psicología Humanística que, a través de incitar a que la Psicología estudie el comportamiento y la mente humana a partir de las capacidades y no solo de las falencias, inaugura de forma explícita el enfoque salugénico en Psicología. Es por eso que, conceptos como el self-actualization de Maslow o el de funcionamiento óptimo de la personalidad de Rogers son antecedentes importantes de la PP. En la década del 80 del siglo $X X$, se comienzan a estudiar una serie de temas que para, algunos investigadores, representa un nuevo paradigma de reflexión teórica y metodológica (Casullo, 2002). Los temas son derivados de la teoría del apego (Bowlby, Ainsworth, Marrone); la creatividad (De Bono, Simonton, Stein, Sternberg, Csikszentmihalyi); la resiliencia (Werner y Smith, Cyrulnik, Melillo); la inteligencia emocional (Goleman); las inteligencias múltiples (Gardner), el bienestar psíquico (Diener, Ryan, Ryyf); entre otros.

En síntesis, aquellos autores y teorías que adscriben a un enfoque salugénico (siendo su más reciente manifestación la PP como se viene mencionando), adhieren a un modelo continuo de funcionamiento humano, en detrimento del categorial (Ver Maddux, 2002; Maddux, Snyder \& Lopez, 2004). Desde este modelo se considera a la experiencia de una persona como una unidad, siendo los extremos del continuo las buenas y malas experiencias. Por lo tanto, todas aquellas intervenciones que sirvan para aminorar lo negativo, también deberían servir para incrementar lo positivo y viceversa (Joseph \& Linley, 2006).
A pesar de lo expuesto, pueden subsistir algunos interrogantes como por ejemplo: ¿por qué se mantuvo vigente por tanto tiempo el modelo enfocado en el malestar? Y, por el contrario, ¿por qué cambiar el enfoque clásico por el de una psicología positiva?

Respondiendo al primer interrogante, Gable y Haidt (2005) proponen tres razones. En primer lugar, la compasión: aquellos que sufren deben ser ayudados antes que los que no lo hacen. En segundo lugar, la preocupación mencionada por recuperar mentalmente a los veteranos afectados por la segunda guerra mundial (Seligman, 2002). Y, en tercer lugar, el hecho de que la investigación sobre aspectos negativos genera un mayor impacto en virtud de que los eventos negativos son menos frecuentes y son procesados más profundamente que los positivos (Baumeister, Bratslavsky, Finkeneauer \& Vohs, 2001; Olson, Roesen \& Zanna, 1996). Sin embargo, estos tres argumentos no aportan la suficiente evidencia empírica para sostener por tanto tiempo la mirada sobre los aspectos negativos de la experiencia humana.

En relación con lo antedicho y respondiendo al segundo interrogante ( ¿Por qué cambiar el enfoque clásico por una psicología positiva?), puede decirse que mucho se ha avanzado en el estudio de las psicopatologías y las psicoterapias, temas quizá influenciados por las guerras mundiales. La psicología ha hecho progresos significativos en el diagnóstico y tratamiento de algunos trastornos psicológicos y se ha ocupado de cómo hacer que las personas sufran menos (Seligman, 1995). Hasta el año 1950 la mayoría de las psicopatologías no eran tratables y no existían acuerdos sobre su delimitación diagnóstica. A principios del siglo XXI existe sólida evidencia empírica de que las psicoterapias funcionan y cuáles son más eficaces para ciertos trastornos (Barret \& Ollendick, 2004; Hibbs \& Jensen, 1996; Kazdin \& Weisz, 2003; Nathan \& Gorman, 2002). Sin embargo, si miramos las tasas de prevalencia de los trastornos depresivos durante el siglo XX es posible apreciar que su frecuencia ha aumentado 10 veces y que de ser una patología adulta en los años sesenta (se desarrollaba alrededor de los 30 años) se ha transformado en una patología adolescente, ya que en la actualidad la edad de inicio es a los 14 años. 
Por otro lado, si miramos los estudios sobre la calidad de vida y el bienestar de las naciones, especialmente para las más desarrolladas, es posible observar que no hubo incrementos significativos en los últimos 40 años (Seligman, 2008). Este cuadro de situación denuncia que no se han hecho esfuerzos para mejorar la calidad de vida de las personas y aquellos dirigidos al tratamiento de las enfermedades mentales no fueron suficientes. El haber centrado el foco en los trastornos psicopatológicos trajo como costo principal que la psicología se transformó en victimología, ocupándose solo de aquellos que requieren tratamiento (alrededor del 20 al 30\% de la población) y dejando de lado el estudio del funcionamiento mental óptimo (Seligman \& Csikszentmihalyi, 2000). Hacer PP no es desconocer que las personas sufren y que se enferman, sino dejar de igualar salud mental con ausencia de enfermedad. Los factores mencionados llevan a la conclusión de que es necesario, no eliminar, sino complementar el modelo médico con los avances en el estudio de los aspectos positivos.

\section{DEFINICIONES DE PSICOLOGÍA POSITIVA}

Habiendo analizado este recorrido desde los inicios de la PP, se está en condiciones de preguntar ¿qué se entiende, entonces, por Psicología Positiva?

Linley, Joseph, Harrington y Wood (2006) destacan una serie de definiciones dadas por referentes de la PP.

El campo de la Psicología Positiva, a un nivel subjetivo, refiere a las experiencias subjetivas: bienestar, alegría y satisfacción (en el pasado); esperanza y optimismo (para el futuro); y fluir -flow- y felicidad (en el presente). A un nivel individual refiere a los rasgos individuales positivos: capacidad de amar, vocación, perseverancia, perdón, originalidad, visión de futuro, espiritualidad, talento, sabiduría. A un nivel grupal, refiere a las virtudes cívicas y las instituciones: responsabilidad, altruismo, moderación, tolerancia, ética (Seligman \& Csikszentmihalyi, 2000, p.5).

La Psicología Positiva no es más que el estudio científico de las fortalezas y virtudes humanas. Analiza al "hombre promedio" con un interés en buscar que funciona, que es correcto, que mejora... (Sheldon \& Kasser, 2001, p. 216).

La Psicología Positiva es el estudio de las condiciones y procesos que contribuyen a prosperar o, al óptimo funcionamiento de personas, grupos e instituciones (Gable \& Haidt, 2005, p. 104).

La Psicología Positiva refiere a las perspectivas científicas acerca de que hace que la vida sea digna de ser vivida. Se centra en los aspectos de la condición humana que llevan a la felicidad, a la completud y a prosperar (The Journal of Positive Psychology, 2005).

Por último, Linley, Joseph, Harrington y Wood (2006) definen a la PP como el estudio científico de las posibilidades de un desarrollo humano óptimo; a nivel meta-psicológico se propone reorientar y reestructurar teóricamente el desequilibrio existente en las investigaciones y prácticas psicológicas, dando mayor importancia al estudio de los aspectos positivos de las experiencias vitales de los seres humanos, integrándolos con los que son causa de sufrimiento y dolor; en un nivel de análisis pragmático, se ocupa de los medios, procesos y mecanismos que hacen posible lograr una mayor calidad de vida y realización personal (p. 8).

Todas estas definiciones contienen temas en común pero también hacen énfasis en cuestiones diferentes. En principio, se debería destacar aquello que no es psicología positiva. En primer lugar, no se trata de un movimiento filosófico ni espiritual, ni son ejercicios de autoayuda para alcanzar la felicidad. Tanto los constructos psicológicos propuestos como la evidencia empírica derivada tienen una sólida base científica. En segundo lugar, la PP no está basada en el criterio de autoridad de su fundador y en la búsqueda de adeptos como hace el saber pseudocientífico, sino que opera a través de la comunidad científica internacional. Las investigaciones derivadas se encuentran publicadas en revistas científicas internacionales de alta calidad. En tercer lugar, no se trata de la negación del sufrimiento y de los aspectos negativos de las personas, sino tender hacia la complementariedad y a corregir el desbalance que sufrió la psicología en los últimos 60 años que otorgó mayor énfasis a los aspectos patológicos de las personas (Castro Solano, 2009). 
La definición dada por Linley y colaboradores sintetiza lo que pretende constituirse como PP. Se podría destacar que todas las definiciones persiguen los siguientes objetivos (Gancedo, 2008): ampliar el foco desde la preocupación casi exclusiva por reparar el daño hacia el fortalecimiento de las potencialidades; investigar el bienestar psíquico y subjetivo; identificar, medir y promover este bienestar en sujetos, grupos y sociedades; y realizar todo lo expuesto con rigurosidad científica. Además Linley y colaboradores resaltan el hecho de que la PP ha provisto de unas "lentes diferentes" para entender la experiencia humana y ha comenzado a fundar un lenguaje compartido para el estudio de los estados y rasgos positivos.

\section{LOS TEMAS DE ESTUDIO: LOS TRES PILARES DE LA PSICOLOGÍA POSITIVA}

A lo largo de este escrito se han venido mencionando algunos temas centrales de la PP, los cuales fueron nucleados en el año 2000 en un número especial de la Revista American Psychologist coordinado por Seligman que tenía como título Psicología Positiva y reunía artículos científicos relacionados con la felicidad, la motivación intrínseca, las ilusiones positivas, la sabiduría, el talento, los mecanismos adaptativos, la creatividad y el desarrollo humano óptimo. Este número fue, como se destacó previamente, uno de los hitos que marcó el inicio de la PP.

En otro de los momentos claves para el despegue de este movimiento (el encuentro entre investigadores realizado en Akumal -México-) se acordaron los pilares básicos de la PP: el estudio de las emociones positivas, de los rasgos positivos y de las instituciones positivas. Por lo tanto, la mayor parte de las investigaciones realizadas hasta el momento recae en el abordaje de alguno de estos tres aspectos.

Algunas de las preguntas que se hacen los investigadores que se enrolan en la PP son: ¿Qué hace que una vida sea plena? Y, ¿Cuáles son los caminos para conseguirla? Según Seligman (2002), existen tres vías de acceso para una vida plena. La primera ruta es a través de las emociones positivas ó dicho en términos del autor: la vida placentera (pleasant life). Esta vía es quizás la más conocida y responde a la noción mas comúnmente extendida de felici- dad. Consiste en incrementar, la mayor parte del tiempo, la cantidad de emociones positivas para ser feliz. La propuesta es netamente hedonista y considera que la felicidad consiste en tener la mayor cantidad de momentos felices a lo largo de la vida. Estos momentos felices pueden alcanzarse en el momento presente a través del savoring y el mindfullness. Ambas consisten en rescatar el momento presente de la forma más genuina posible. El savoring, o saborear el momento presente, consiste en deleitar los sentidos de la forma más auténtica posible. Por el otro lado, el mindfullness es un estado mental que se alcanza a través del entrenamiento caracterizado por la atención plena de los propios pensamientos, acciones o sensaciones. Las emociones positivas también pueden alcanzarse focalizándose en el pasado al cultivar la gratitud y el perdón o, en el futuro a través de la esperanza y el optimismo. Este acceso a la felicidad (vida placentera) tiene dos límites importantes. En primer lugar, el nivel de emocionalidad positiva está muy ligado a la personalidad y los componentes genéticos heredables (entre un $40 \%$ a un $50 \%$ ). Esto significa que el nivel de emocionalidad positiva que tienen las personas es más o menos fijo, con poca probabilidad de cambio (Lyubomirsky, 2007). En segundo lugar, otra desventaja es que ocurre un fenómeno que se denomina adaptación hedónica o habituación. Las personas se habitúan rápidamente a las emociones positivas y resulta necesario incrementar el monto de estas emociones de forma permanente hasta un punto tal en el que el incremento no causa mayor felicidad. Sería un límite autoimpuesto para el placer sin límites que tiene su origen en nuestro pasado evolutivo. Operaría como un factor protector frente a las adversidades.

Está demostrado científicamente que si bien las personas reaccionan con alta emocionalidad negativa a los eventos vitales estresantes luego de un tiempo vuelven a una línea base (set point) sin importar la intensidad del evento (Diener, Lucas \& Scollon, 2006). Esto solo es posible gracias al mecanismo de habituación, si se experimentasen grandes cantidades de afecto positivo, se experimentarían grandes cantidades de afectos negativos también.

Afortunadamente las emociones positivas no son la única vía de acceso hacia la vida plena. En segundo lugar, se encuentra la ruta que tiene 
que ver con el compromiso (engagement life). El placer se deriva del compromiso con la tarea efectiva y con la capacidad de experimentar flow. Se alcanza el estado de flow cuando una actividad absorbe al sujeto y tiene la sensación de que el tiempo se detuvo. Tal vez pueda alcanzarse el estado de flow mediante algún desafío en el trabajo, la lectura de un libro o al tocar un instrumento. Para alcanzar el flow es necesario que la tarea no sea ni muy aburrida (porque el estado de flow no se alcanza) ni tampoco muy estresante, porque el flow se pierde. Es una ecuación entre las habilidades personales y las características de la tarea. Si se es conciente de las fortalezas personales al aplicarlas en una tarea concreta se alcanza esa conexión tan particular denominada compromiso (engagement). El flow no se da de primera mano como en las emociones positivas, sino que exige esfuerzo y trabajo.

Mientras que las dos primeras rutas hacia la felicidad tienen que ver con cuestiones individuales sin importar demasiado el entorno, la tercer vía de acceso o vida con significado (meaningfull life), tiene que ver con la búsqueda de sentido y eso solo puede entenderse en un contexto global más amplio. Esta tercera vía es la aplicación de las fortalezas personales para el desarrollo de algo más importante y amplio que uno mismo. Es poder aplicar las fortalezas para ayudar a los demás y hacer que éstos puedan desarrollar sus potencialidades. Generalmente este proceso se da a través de las instituciones: la familia, el trabajo, la escuela, la comunidad. En suma, es poner las capacidades al servicio de los demás para obtener significado vital como resultado de este proceso.

Las tres rutas mencionadas son complementarias, si bien las personas pueden alcanzar la vida plena a través de las tres vías, aquellos que registran mayor satisfacción son los que emplean conjuntamente las tres rutas (Peterson, 2006). En términos de la psicología positiva, la vida placentera da lugar al estudio de las emociones positivas; la vida comprometida está relacionada con el estudio de las fortalezas, las virtudes personales, y los estados de flow y la tercera está en relación con las instituciones positivas, siendo este último tópico el que está conceptualmente menos desarrollado. Los autores del campo de la psicología positiva han derivado instrumentos para evaluar los tres componentes de la vida plena y aseveran que también son entrenables, mediante intervenciones específicas (Seligman, Steen, Park \& Peterson, 2005; Seligman, Rashid \& Parks, 2006). Recientemente Seligman (2009) agregó un cuarto pilar de estudio: los vínculos positivos (la vida social) como vía de acceso a la felicidad y que tiene su origen en las investigaciones sobre el bienestar psicológico que identifican a las personas extremadamente sociables como las más felices.

\section{LOS INVESTIGADORES, LA PRODUCCIÓN Y LA DIFUSIÓN}

Seligman es el fundador y principal referente de la PP, seguido por los miembros que integran el Comité de PP: Csikszentmihalyi, Diener, Jamieson, Peterson y Vaillant. Los tres primeros dirigen cada uno un Centro especializado en alguno de los tres pilares de la PP: Diener dirige el abocado al estudio de las emociones positivas, Csikszentmihalyi el de los rasgos positivos, y Jamieson el de las instituciones positivas. También es importante el trabajo de investigadores como Snyder, Frederickson, Haidt, Segerstrom y todos los autores que han sido mencionados a lo largo de este escrito que se han abocado a alguno de los diferentes tópicos que aborda la PP.

Es llamativo el crecimiento que ha tenido la PP desde su surgimiento y que se ve reflejada en un importante cúmulo de producción como se detalla más adelante. Para lograr este rápido avance fue valioso el aporte financiero dado por instituciones como la Gallup Organization, la Templeton Foundation, la Mayerson Foundation y la Atlantic Philantrophies, entre otras.

Los investigadores nombrados publican sus trabajos en las revistas científicas más importantes y asimismo fundaron otras nuevas con el propósito de publicar trabajos inéditos en el área. En América latina, Psicodebate es pionera en esta tarea. En los Estados Unidos American Psychologist, la revista oficial de la APA, ha publicado números especiales y secciones de artículos referentes a la psicología positiva. The Journal of Happiness Studies, The Journal of Positive Psychology and The Journal of Health and Well-being, son revistas científicas creadas con el propósito de publicar artículos sobre psicología positiva. 
Los libros sobre psicología positiva comenzaron a aparecer con increíble rapidez. Algunos de ellos son de corte académico escritos por especialistas y otros están dirigidos a una audiencia más amplia. Sin importar el tipo de público, los autores tienen especial cuidado en que el contenido de los libros tenga una sólida base empírica. Desde la publicación del libro de Seligman Auténtica Felicidad en el 2002, otras publicaciones destacables son: Character Strengths and Virtues: A Handbook and Classification (Peterson \& Seligman, 2006). Este se propone como un Manual de Sanidades que sea la contraparte del DSM y la CIE al establecer un vocabulario común sobre rasgos positivos evaluables. Consiste en una clasificación de seis virtudes (e.g. sabiduría) compuesta cada una por una serie de fortalezas que la definen (e.g. creatividad, curiosidad, apertura mental, amor por el saber, perspectiva). Otros libros reconocidos son: The Psychology of Gratitude (Emmons \& McCullough, 2003), The Happiness Hypothesis (Haidt, 2006), A Primer in Positive Psychology (Peterson, 2006), Positive Psychology: The Scientific and Practical Explorations of Human Strengths (Snyder \& Lopez, 2007), The How of Happiness (Lyubomirsky, 2007), Happiness: Unlocking the Mysteries of Psychological Wealth (Diener and Biswas-Diener, 2008), y más recientemente Positivity (Fredrickson, 2009).

Los libros de referencia en el área incluyen: A Handbook of Positive Psychology (Snyder \& Lopez, 2002), Positive Psychology in Practice (Linley \& Joseph, 2004), y la Encyclopedia of Positive Psychology (Lopez, 2008). Además de estos libros en inglés, se han publicado otras obras en idioma español: Evaluación del Bienestar Psicológico en Iberoamérica (Casullo, 2002), Prácticas en Psicología Positiva (Casullo, 2008), Psicología Positiva Aplicada (Vázquez \& Hervás, 2008) y La ciencia del bienestar. Fundamentos para una psicología positiva (Vázquez \& Hervás, 2009). (Para un listado más completo de libros visite el sitio web de la Universidad de Pennsylvania: Positive Psychology Center: www.positivepsychology.org).

También es de destacar la expansión que han tenido los programas educativos en psicología positiva. El primer programa con título de posgrado abrió sus puertas en la Universidad de Pennsylvania en otoño de 2005. Este Master en Psicología Positiva Aplicada es un programa intensivo dirigido a profesionales en las áreas de educación, derecho, medicina, economía y negocios que buscan aprender de forma mas profunda la teoría y la práctica de la psicología positiva para poder aplicarla en sus campos específicos de trabajo. Está también dirigido a jóvenes estudiantes que quieren aprender acerca de la psicología positiva antes de iniciar un programa de doctorado u otro título superior de posgrado. En el 2007 la University of East London en el Reino Unido lanzó un programa de psicología positiva aplicada y en el mismo año la Claremont Graduate University en los Estados Unidos abrió su master y programa de doctorado en psicología positiva. En el 2008 la Universidad Iberoamericana en la ciudad de México abrió el primer programa de posgrado en el área, en español. Australia, Israel, Italia y Portugal están desarrollando programas de posgrado en psicología positiva. Los graduados de estos programas están comenzando a aplicar la psicología positiva en el campo profesional de modos novedosos, trabajando para transformar los individuos, escuelas, empresas y otras organizaciones a la luz de la teoría y la práctica de la psicología positiva. El Center for Applied Positive Psychology en el Reino Unido es una organización sin fines de lucro desarrollada por Alex Linley para fomentar la aplicación de la psicología positiva en varias áreas.

El desarrollo de los sitios web resulta también clave y han sido traducidos al español tales como www.authentichappiness.org que ofrece sin costo alguno la administración y evaluación de importantes instrumentos de constructos derivados de la psicología positiva, y www. es.pos-psych.com que cuenta con interesantes artículos sobre la aplicación de la psicología positiva.

Asimismo, se han fundado algunas organizaciones académicas para expandir la misión de la psicología positiva. Entre estas se pueden incluir la European Network of Positive Psychology, la Società Italiana di Psicologia Positiva, y las más recientemente creada International Positive Psychology Association (IPPA). La misión de la IPPA es "promover la ciencia y la práctica de la psicología positiva y facilitar la comunicación y colaboración entre los investigaciones y profesionales de todo el mundo que estén interesados en la psicología positiva". 
La IPPA se fundó en 2007 con los líderes más importantes de la psicología positiva en todo el mundo. Entre ellos se destacan Seligman, Csikszentmihalyi, Diener, Peterson, Grant, Veenhoven, Delle Fave, Vázquez, y Casullo. Se estableció un sitio web (www.ippanetwork. org) y se inauguraron una serie de teleconferencias, un newsletter mensual para informar de los eventos y las investigaciones en psicología positiva a lo largo y ancho del planeta. IPPA también está organizando una red iberoamericana en psicología positiva, se trata de una red en idioma español que se especializa en temas de psicología positiva en América Latina y en la península ibérica. En el año 2009 esta asociación organizó el primer congreso internacional de psicología positiva en la ciudad de Filadelfia, Pennsylvania.

\section{IMPLICANCIAS PRÁCTICAS DE LA PSICOLOGÍA POSITIVA: LAS INTERVENCIONES}

A partir de lo mencionado hasta aquí se desprende que la PP no debe solo preocuparse por describir las emociones, los rasgos y las instituciones positivas sino, también, por tratar de lograr que se den y/o que se incrementen. Por lo tanto, unos de los intereses principales recae en la investigación acerca de las intervenciones positivas, por ejemplo, para incrementar la felicidad (Seligman, Steen, Park \& Peterson, 2005). Una de las razones por las cuales es importante incrementar la felicidad es el hecho que se ha revelado hace unos pocos años que la felicidad es causal y que trae aparejado muchos beneficios, como por ejemplo, sentirse más saludable, exitoso y más comprometido socialmente (Lyubomirsky, King \& Diener, 2005). Seligman, Steen, Park y Peterson (2005) probaron la eficacia de un método consistente en cinco ejercicios (visitas de gratitud, escritura de tres cosas buenas de la vida, escritura de experiencias de éxito, usar las fortalezas de un modo distinto, identificar y usar las principales fortalezas) para incrementar la felicidad hallando resultados promisorios en cuanto a efectos tanto de corto como de largo plazo.

Las intervenciones que se realizan en el campo de la psicología positiva no se hacen desde el modelo médico de salud-enfermedad ya que eliminar lo negativo no siempre trae como consecuencia un cambio positivo (Keyes \& López, 2002). Las psicoterapias deberían proveer de coraje, insight, optimismo, honestidad y perseverancia y estructurar una habilidad en las personas que opere como barrera frente a los estresores y dificultades futuras. Una psicoterapia positiva estaría encaminada hacia la mejora de los rasgos positivos, el cultivo de las fortalezas humanas y la ayuda que se da a los clientes para estimular el cambio positivo (Seligman, 2002). Se caracteriza porque el paciente en este tipo de intervenciones es fundamentalmente activo, ya que es a través de las actividades intencionales que se alcanza el cambio. Las intervenciones de la PP si bien son relativamente nuevas tienen sus antecedentes en la psicología de counseling de los años 50 en las terapias denominadas del crecimiento personal o del potencial humano. Otros tratamientos positivos por fuera del paraguas de la psicología positiva también se encuentran en esta línea, tales como las terapias focalizadas en soluciones, las terapias de clarificación de valores y las basadas en la teoría de la esperanza, entre otras. Según Pawelski (2009), la intervención debe ser positiva tanto en punto de aplicación como en método. Debe estar dirigida a aumentar el bienestar (punto de aplicación) pero a través de los tres pilares de la PP (mejora de afecto positivo, aplicación de fortalezas y cultivo de significado).

En la última década hubo una proliferación de programas de intervenciones de PP, dirigidos fundamentalmente a mejorar el bienestar de las personas. La mayoría de ellos no están basados en una base teórica integradora, sino que se basan en microteorías o en datos empíricos de líneas de investigación sencillas (Vazquez, Sánchez \& Hervás, 2008). La estrategia empleada consiste en detectar las variables psicológicas que diferencian a los individuos con alto y bajo bienestar mediante estudios correlacionales y longitudinales $y$, luego, se verifica la eficacia de las intervenciones de forma experimental en diversos grupos de pacientes y en población general no clínica, tal como el ejemplo mencionado al inicio de este apartado sobre como incrementar la felicidad. Sin embargo, uno de los programas pioneros para elevar la felicidad de las personas fue el de Fordyce $(1977,1983)$, quien utilizó una metodología muy cuidada para valorar la efectividad de las intervenciones diseñadas. Su programa consistía 
de 14 actividades entre las que se contaba el empleo en actividades sociales, el desarrollo del pensamiento positivo, el empleo de actividades físicas, la conservación de relaciones íntimas, entre otras. El programa de Fordyce duraba dos semanas y demostró ser eficaz para elevar la felicidad medida por un instrumento creado a tal efecto. Fordyce demostró que la felicidad podía ser modificada mediante intervenciones específicas y que era necesario el uso de actividades intencionales sostenidas de los participantes para elevar dicho nivel.

Los estudios de Pennebaker y Segal (1999) sobre la escritura de sucesos traumáticos demuestran que esta técnica narrativa permite elaborar dichos eventos. Burton y King (2002) demostraron experimentalmente que la escritura de hechos positivos mejora las emociones positivas y por ende eleva el bienestar. Estas investigaciones verificaron que la utilización de técnicas narrativas provoca un aumento de las emociones positivas y un menor riesgo de enfermedad en los meses siguientes de instrumentado el protocolo.

En la misma línea Lyubomirsky (2007) diseñó una serie de actividades con el propósito de mejorar el bienestar. Estas tareas estaban basadas en contar cosas buenas que les habían sucedido a las personas, escribir cartas de gratitud, visualizar el mejor self futuro mediante técnicas narrativas y utilizar técnicas de saboreo (savoring) respecto de los buenos momentos. En todos los casos los grupos experimentales a los que se les instruyeron las tareas obtenían ganancias significativas en los niveles de bienestar psicológico.

Bono y Mc Cullough (2006) demostraron que el incremento de las experiencias de gratitud está asociado con el bienestar psicológico. Las investigaciones de intervenciones trataron de inducir experimentalmente actividades de gratitud mediante técnicas escritas (e.g. escriba una carta para alguien con el que se siente agradecido) o instrucción de actividades específicas (e.g. acciones de gratitud reales) demostrando, en todos los casos, un mayor aumento de bienestar de las personas (Lyubomirsky, Sheldon \& Schakade, 2005; Emmons \& Mc Cullough, 2003; Watkins, Woodward, Stone \& Kolts, 2003). Al respecto Seligman (2003) comenta que el motivo por el cual la gratitud mejora el bienestar es porque amplía los buenos recuerdos sobre el pasado en cuanto a su intensidad y frecuencia, así como al etiquetado de los pensamientos, amplificando los recuerdos positivos.

Otras intervenciones basadas en la hope theory (Snyder, 2002) estuvieron dirigidas hacia la activación del componente esperanza. Este modelo plantea como elemento fundamental el logro de metas, teniendo en cuenta además las expectativas del futuro, la motivación y la planificación necesarias para conseguir dichos objetivos. Los trabajos de Irving, et.al. (2004) demostraron, en un formato de tratamiento breve focalizado en la esperanza (antes del tratamiento clínico propiamente dicho), la eficacia de intervenciones dirigidas a la esperanza en un grupo de pacientes clínicos. Otra investigación que tenía como objetivo verificar la eficacia de intervenciones basadas exclusivamente en la esperanza demostraron que las actividades relacionadas con la formulación de metas, el uso de vías múltiples para alcanzar los objetivos, la utilización de recursos motivacionales y la monitorización de progresos era muy útil para mejorar la esperanza (Cheavens, Feldman, Woodwaard \& Snyder, 2006).

Fava (1999) diseñó un programa de intervenciones positivas para promover el bienestar de modo complementario a otros tratamientos farmacológicos o psicológicos. El programa se basaba en el reconocimiento de recuerdos de eventos positivos y en la identificación de obstáculos que obstaculizan dichos recuerdos. Tiene como base teórica la teoría del bienestar de Ryff (1989). Se ha demostrado la eficacia de esta intervención en pacientes depresivos y con trastornos de ansiedad generalizada (Fava \& Ruini, 2003). En todos los casos el módulo de terapia del bienestar, combinada con otro tratamiento clínico, potencia la eficacia de los resultados de las terapias convencionales.

Frisch $(1998,2006)$ plantea un tipo de intervención clínica llamada terapia de calidad de vida, que integra una teoría del bienestar, instrumentos de medida específicos y recursos de intervención especialmente diseñados para cultivar aspectos positivos y aumentar el bienestar. Si bien esta terapia es prometedora porque integra un modelo completo de intervención, aun no tiene suficiente validación empírica que avale sus resultados (Vázquez, Sánchez \& Hervás, 2008). 
Fredrickson $(2001,2009)$ propone una teoría de las emociones positivas que se denomina de ampliación y construcción. La autora demostró, con firme evidencia empírica, que si las personas incrementan su emocionalidad positiva mediante diferentes técnicas de forma sostenida (e.g., meditación, savoring, flow) las personas, no solamente se vuelven más creativas y resuelven mejor los problemas que se les presentan (efecto ampliación), sino que las emociones positivas en el largo plazo crean recursos personales y resiliencia (efecto construcción). Como técnica de intervención recomienda la creación de portfolios personalizados de emociones positivas para ser usados de forma alternada con el fin de evitar la adaptación hedónica. Así, por ejemplo, si se quiere estimular la emoción alegría, se debe ubicar en una caja, pizarra o archivo de computadora todo tipo de fotos, objetos, videoclips, esencias, recuerdos, etc., que evoquen momentos alegres, debiéndose trabajar con todo el espectro emocional de la teoría.

Como se puede apreciar existe una gran cantidad de programas de intervención basados en los fundamentos de la PP lo cual delimita un prolífero campo de investigación y aplicación.

\section{DISCUSIÓN FINAL: LAS CRÍTICAS A LA PSICOLOGÍA POSITIVA Y LAS PERSPECTIVAS FUTURAS}

Según los diferentes autores que se aborde, la PP se presenta como una nueva ciencia, un nuevo paradigma, un nuevo movimiento o una nueva corriente (Gancedo, 2008). Todos los términos no están exentos de críticas siendo los dos primeros demasiado ambiciosos y los últimos dos demasiado flexibles y ambiguos.

Más allá del calificativo con el que se la nombre, la PP ha recibido otra serie de críticas que son importantes tener en cuenta. Una de las primeras objeciones es que la PP no aporta novedad alguna al quehacer del psicólogo. Muchos autores en la historia de la psicología han trabajado con constructos salugénicos, por lo tanto la hipotética fundación de Seligman no sería un inicio genuino. El aporte de Seligman es quizá el haber reunido en un gran paraguas diversas líneas de investigación y autores que trabajaban de modo atomizado en temas positi- vos y el haber cristalizado en un movimiento diversas corrientes enroladas dentro del enfoque salugénico, poniendo en el centro la temática del bienestar psicológico (Gancedo, 2009). Sin embargo, la PP aún adeuda la construcción de un modelo epistemológico unificado que de cuenta del bienestar, ya que se observa un solapamiento de constructos y conceptos teóricos en lugar de un paradigma unificado y consistente.

Además, se suma el distanciamiento que proponen sus fundadores respecto de los antecedentes históricos de la PP relacionados con la psicología humanista. Si bien este enfoque es un antecedente importante de la PP, esto solo ha sido considerado solo de modo reciente (Peterson, 2006). Tanto Seligman como Csikszentmihalyi intentaron distanciarse de este enfoque haciendo un fuerte énfasis en el método científico que los autores positivos emplean como un modo de validar sus afirmaciones y hallazgos. Sin embargo, conceptos tales como el sentido de la vida, el proyecto vital, el estudio de la potencialidades psíquicas y los aspectos sanos del ser humano como elementos centrales de la psicología humanística están muy próximos a las propuestas de la PP. En la misma línea, otros modelos salugénicos de mediados de los años 80 con sólida base empírica tales como el propuesto por Antonovsky (1988) o por Kobaza (1979) no son ni siquiera considerados como antecedentes del movimiento.

Otra crítica consiste en que la PP no agrega nada al sentido común de cómo vivir bien la vida y cuál es el camino para alcanzarla. Sin embargo, muchos hallazgos de la PP son en verdad contraintuitivos (Peterson, 2006). La gente piensa que lucir bien, ser inteligente y tener dinero son las claves del bienestar. Sin embargo, las tres últimas décadas de estudio sobre la satisfacción han arrojado resultados descorazonadores. El éxito económico, la inteligencia y el atractivo físico son variables que explican poco menos del $10 \%$ de la varianza del bienestar psicológico (Diener, 1994). La gente asimismo piensa que trabajando y elaborando situaciones del pasado o circunstancias presentes se alcanzará la felicidad, sin embargo la PP ha demostrado de forma reiterada que un cambio de las condiciones presentes no trae aparejado un incremento significativo en la felicidad de las personas (Lyubomirsky, 2007). 
En relación a estos aspectos debe tenerse en cuenta que la PP puede correr un peligro muy importante y que consiste en abandonar la actitud descriptiva de la ciencia por una actitud prescriptiva propia de los sistemas morales. Cabe destacar que lo que resulta positivo en un contexto o ambiente puede no serlo en otro o, no acarrear las mismas consecuencias positivas, por lo cual resulta demasiado ambicioso y complejo sugerir "que es bueno" (Aspingwall \& Staudinger, 2003, Gable \& Haidt, 2005; Held, 2004; Linley, Joseph, Harrington, 2006; Taylor, 2001).

Por otro lado, en los ámbitos académicos muchas veces la PP no es considerada seria. Temas relacionados con el bienestar serían considerados en extremo superficiales y poco serios como para ser investigados o tomados en cuenta en los ámbitos académicos. El estudio de la felicidad estaría mas en relación con los libros de autoayuda o los cursos de crecimiento personal. Sin embargo, cuatro décadas de investigación sobre el bienestar ha puesto de manifiesto hallazgos empíricos de relevancia que aportan las claves de la felicidad de las personas. Actualmente son la base sobre la que se asientan las modernas intervenciones positivas como las que se mencionaron en el apartado anterior (Lyubomirsky, King, \& Diener, 2005).

Por todo lo antedicho se desprende que a la PP le resta mucho por avanzar y consolidar, hasta el momento ha abierto un abanico de tópicos que necesitan ser abordados científicamente. En palabras de Gable y Haidt (2005) la PP debe ayudar a comprender los factores que generan fortalezas y delimitar como éstos contribuyen a la salud física, el bienestar subjetivo, el contacto grupal e institucional, delineando, además, intervenciones efectivas que favorezcan a estos procesos. No se intenta eliminar los avances logrados por el modelo médico sino intentar una integración con los desarrollos de la $\mathrm{PP}$, de manera que las intervenciones positivas sean un complemento de las terapias basadas en problemas (Linley, Joseph, Harrington \& Wood, 2006; Seligman, Steen, Park \& Peterson, 2005; Sternberg \& Grigorenko, 2001). Ya desde el inicio, Seligman (2003) delimitó los temas que se estaban abordando y los que restaban estudiar como, por ejemplo, no solo como medir el bienestar subjetivo individual sino también la necesidad de analizar lo que el denomina bienestar colectivo; o que aportes pueden realizar las neurociencias (como, por ejemplo, explicar factores hereditarios) para explicar los estados positivos. Además de estas cuestiones, resulta perentorio el abordaje del tercer pilar de la PP: las instituciones positivas, ya que como se mencionó previamente es el menos desarrollado (Gable \& Haidt, 2005).

Es indudable que la PP como movimiento ha venido a corregir el desbalance en la psicología entendida como psicología clínica, haciendo énfasis en los aspectos positivos del ser humano. Muchos autores sostienen que a mas de una década de su existencia la PP se plantea una verdadera encrucijada: o bien se propone como una especialidad más de la psicología, o bien se integra con otros campos del quehacer profesional del psicólogo.

Casullo (2008) comenta que si lo hace tendrá un futuro promisorio, sino permanecerá marginal y fragmentada. Al respecto Lazarus (2003) comenta que el éxito de la psicología positiva certificará su desaparición dejándonos una psicología balanceada que tome en cuenta tanto aspectos negativos como positivos. Tal como sostiene Vazquez (2006), la psicología positiva del futuro será psicología o no será nada. Tal vez su nacimiento tenga que ver con su temprana disolución como movimiento.

Quizá este sea el mejor destino y la psicología positiva habrá cumplido con su cometido, su desaparición será en este caso una certificación de su éxito: será una empresa formidable para la Psicología contribuir a desarrollar un esfuerzo programático en la consolidación teórica de los conceptos más relevantes y las relaciones entre los mismos (emociones positivas, bienestar, salud positiva, etc.), el desarrollo de herramientas de evaluación válidas de tales conceptos (¿cómo medimos, por ejemplo, el grado de bienestar de un individuo?) y, finalmente, la exploración y análisis de vías de intervención que promuevan o ayuden a mantener el grado de bienestar de los ciudadanos.

\section{REFERENCIAS}

Allport, G.W. (1958). Becoming: Basic consideration for psychology of personality. Oxford, England: Verlag. 
Antonovsky, A. (1979). Health, Stress and Coping: New Perspectives on Mental and Physical Well-being. San Francisco: Jossey-Bass.

Antonovsky, A. (1987). Unraveling the mystery of health. How people manage stress and stay well. San Francisco: Jossey-Bass.

Antonovsky, A. (1988). Unravelling the mystery of health. San Francisco: Jossey-Bass.

Aspingwall, L.G. \& Staudinger, U.M. (2003). A psychology of human strengths: some central issues of an emerging field. En L.G. Aspingwall. \& U.M. Staudinger (Eds.), A psychology of human strengths: Fundamental questions and future directions for a positive psychology (pp. 9-22). Washington, DC: EEUU: APA.

Barret, P. \& Ollendick, T. (2004). Handbook of interventions that work with children and adolescents: prevention and treatment. West Sussex, UK: Wiley.

Baumeister, R.F., Bratlasvsky, E. Finkenauner \& Vohs, K.D. (2001). Bad is stronger than good. Review of General Psychology, 5, 323-370.

Bono, G. \& McCullough M. E. (2006). Positive responses to benefit and harm: Bringing forgiveness and gratitude into cognitive psychotherapy. Journal of Cognitive Psychotherapy, 20, 147-158.

Burton, C. M. \& King, L.A. (2004). The health benefits of writing about intensely positive experiences. Journal of Research in personality, 38, 150-163.

Castro Solano, A. (2009). Psicología Positiva: ¿una nueva forma de hacer psicología?. Manuscrito enviado para su evaluación.

Casullo, M.M. (2002). Psicología salugénica o positiva. Algunas reflexiones. Anuario de investigaciones. Facultad de Psicología. Universidad de Buenos Aires, 8.

Casullo, M.M. (2003). Evaluación del bienestar psicológico en Iberoamérica. Buenos Aires: Paidós.

Casullo, M.M. (2008). Prácticas en psicología positiva. Buenos Aires: Lugar.

Csikszentmihalyi, M. (2003). Legs or wings? A reply to R.S. Lazarus. En Comentaries on "Does the Positive Psychology movement have legs?" En Psychological Inquiry, 14, 2, 110-172.

Deci, E.L. \& Ryan, R.M. (1985). Intrinsic motivation and self-determination in human behavior. New York: Plenum Press.

Diener, E. (1994). Assessing subjective well-being: Progress and opportunities. Social Indicators Research, 31, 103-157.

Diener, E., \& Biswas-Diener, R. (2008). Happiness: Unlocking the Mysteries of Psychological Wealth. Malden, MA: Blackwell Publishing.

Diener, E., Lucas, R. E., \& Scollon, C. N. (2006). Beyond the hedonic treadmill: Revising the adaptation theory of well-being. American Psychologist, 61, 305-314.

Emmons, R.A., \& McCullough, M.E. (2003). Counting blessings versus burdens: Experimental studies of gratitude and subjective well-being. Journal of Personality and Social Psychology, 84, 377-389.
Erikson, E. (1963). Childhood and society ( $2^{\text {nd }}$ ed.). New York: Norton.

Erikson, E. (1982). The life cycle completed. New York: Norton.

Fava, G. (1999). Well being therapy: Conceptual and technical issues. Psychotherapy and Psychosomatics, 68, 171-179.

Fava, G. \& Ruini, C. (2003). Development and characteristics of a well-being enhancing psychotherapeutic strategy: Well-being therapy. Journal of Behavior Therapy and Experimental Psyquiatry, 34, 45-63.

Fordyce, M. W. (1977). Development of a program to increase happiness. Journal of Counseling Psychology, 24, 511-521.

Fordyce, M. W. (1983). A program to increase happiness: Further studies. Journal of Counseling Psychology, 30, 483-498.

Fredrickson, B. L. (2001). The role of positive emotions in positive psychology: The broaden-and-build theory of positive emotions. American Psychologist, 56, 218226.

Fredrickson, B. L. (2009). Positivity. New York: Crown Publishers.

Frisch, M. B. (1998). Quality of life therapy and assessment in health care. Clinical Psychology. Science and Practice, 5, 19-40.

Frisch, M. B. (2006). Quality of life therapy: Applying a life satisfaction approach to positive psychology and cognitive therapy. Hoboken, NJ: Wiley.

Gable, S.L. \& Haidt, J. (2005). What (and why) is positive psychology? Review of General Psychology, 9, 103-110.

Gancedo, M. (2008). Historia de la psicología positiva. Antecedentes, aportes y proyecciones. En M.M. Casullo (Ed.), Prácticas en psicología positiva. Buenos Aires: Lugar.

Gancedo, M. (2009). Psicología Positiva: posible futuro y derivaciones clínicas. Psicodebate, 9, 15-26.

Godoy, J.F. (1999). Psicología de la Salud: delimitación conceptual. En M.A. Simón (Ed.), Manual de Psicología de la Salud: Fundamentos, Metodología y Aplicaciones (pp. 39-65). Madrid: Biblioteca Nueva.

Haidt, J. (2006). The Happiness Hypothesis. New York: Basic Books.

Held, B.S. (2004). The negative side of positive psychology. Journal of Humanistic Psychology, 44, 9-46.

Hibbs, E. \& Jensen, P. (1996). Psychosocial treatments for child and adolescent disorders: empirically based strategies for clinical practice. Washington, DC, US: American Psychological Association.

Irving, L.M., Snyder, C. R., Cheavens, J., Gravel, L., Hanke, J. Hillberg, P., et al. (2004) The relationship between hope and outcomes at the pretreatment, beginning and later phases of psychotherapy. Journal of Psychotherapy Integration, 14 (4), 419-443.

Jahoda, M. (1958). Current concepts of positive human health. New York: Basic Books.

James, W. (1902). The varieties of religious experience: $A$ study in human nature. New York: Longman, Green. 
Kazdin, A. \& Weisz, J. (2003). Evidence based psychotherapies for children and adolescents. New York: Guilford.

Keyes, C. L. M., \& Lopez, S. J. (2002). Toward a science of mental health: Positive directions in diagnosis and interventions. In C. R. Snyder \& S. J. Lopez (Eds.), Handbook of positive psychology (pp. 45-59). London: Oxford University Press.

Kobasa, S. (1979). Stressful life events, personality and health: An inquiry into hardiness. Journal of Personality and Social Psychology, 37, 1-11.

Lazarus, A. (2003). Does the positive psychology movement have legs? Commentaries on "Does the positive psychology movement have legs?" Psychological Inquiry, 14, (2), 33-52.

Linley, A., Joseph, A., Harrington, S. \& Wood, A. (2006). Positive psychology: Past, present and (possible) future, The Journal of Positive Psychology, 1, (1), 3-16.

Linley, P.A. \& Joseph, S. (2004). Positive Psychology in practice. New Jersey: John Wiley \& Sons.

Lopez, S. (2008). Encyclopedia of Positive Psychology. New York: Wiley-Blackwell.

Lyubomirsky, S. (2007). The how of happiness. New York: The penguin press.

Lyubomirsky, S., King, L., \& Diener, E. (2005). The benefits of frequent positive affect: Does happiness lead to success? Psychological Bulletin, 131, 803-855.

Lyubomirsky, S., Sheldon, K. M., \& Schkade, D. (2005). Pursuing happiness: The architecture of sustainable change. Review of General Psychology, 9, 111-131.

Maddux, J.E. (2002). Stopping the madness: Positive Psychology and the deconstruction of the illness ideology and the DSM. In C.R. Snyder \& S.J. Lopez (Eds.), Handbook of positive psychology (pp. 13-25). New York: Oxford university Press.

Maddux, J.E., Snyder, C.R. \& Lopez, S.J. (2004). Toward a positive clinical psychology: Deconstructing the illness ideology and constructing an ideology of human strengths and potential. In P.A. Linley \& S. Joseph (Eds.), Positive psychology in practice (pp. 230-334). Hoboken, N.J.: Wiley.

Maslow,A.H. (1954). Motivation and personality. New York: Harper.

Maslow, A.H. (1968). Toward a psychology of being. New York: Van Nostrand.

Nathan, P. \& Gorman, G. (2002). A guide to treatments that work. New York: Oxford University Press.

Olson, J.M., Roese, N.J. \& Zanna, M.P. (1996). Expectancies. In E.T. Higgins \& A.W. Kruglansky (Eds.), Social psychology: Handbook of basic principles (pp. 211-238). New York: Guilford Press.

Pawelski, J. (2009, junio). Positive interventions: Theory, research and practice. Workshop. Documento presentado en First World Congress on Positive Psychology. International Positive Psychology Association, Philadelphia, EE.UU.

Pennebaker, J.W. \& Seagal, J.D. (1999). Forming a story: the health benefits of narrative. Journal of Clinical Psychology, 55, 1243-54.
Peterson, C. \& Seligman, M. (2006). Character Strengths and Virtues: A Handbook and Classification. New York: Oxford University Press.

Peterson, C. (2006). A primer in positive psychology. New York: Oxford University Press.

Real Academia Española (2001). Diccionario de la Lengua Española. Vigésima segunda edición.

Rogers, C.R. (1961). On becoming a person: A therapist's view of psychotherapy. Boston: Houghton Mifflin.

Ryff, C. (1989). Happiness is everything, or is it? Explorations on the meaning of psychological well-being. Journal of Personality and Social Psychology, 57 (6), 1069-1081.

Ryff, C.D. \& Singer, B. (1996). Psychological well-being: Meaning, measurement and implications for psychotherapy research. Psychotherapy and Psychosomatics, 65, 14-23.

Salvat Ed. (1976). Diccionario terminológico de ciencias médicas. Undécima edición. Barcelona: Salvat.

Seligman, M. (1995).The effectiveness of psychotherapy: The Consumer Reports Study, American Psychologist, 50, 12, 965-974.

Seligman, M. (2002). La auténtica felicidad. Barcelona: Vergara.

Seligman, M. (2003). Positive psychology: Fundamental assumptions. American Psychologist, 126-127.

Seligman, M. (2008, junio). Positive psychology leader series. Documento presentado en Conference of International Association of Positive Psychology, Philadelphia, EE.UU.

Seligman, M. (2009, junio). Special Lecture. Documento presentado en First World Congress on Positive Psychology. International Positive Psychology Association, Philadelphia, EE.UU.

Seligman, M. \& Csikszentmihalyi, M. (2000). Positive psychology: An introduction, American Psychologist, 55, 5-14.

Seligman, M., Rashid, T. \& Parks, A. (2006). Positive psychotherapy. American Psychologist, 774-788.

Seligman, M., Steen, T., Park, N. \& Peterson, C. (2005). Positive psychology progress, American Psychologist, 60, 5, 410-421.

Seligman, M.E.P. (1999). The president's address. American Psychologist, 54, 559-532.

Sheldon, K.M. \& Kasser, T. (2001). Goals, congruence and positive well-being. New empirical support for humanistic theories. Journal of humanistic psychology, $41,30-50$.

Snyder, C. R. (2002). Hope theory: Rainbows of the mind. Psychological Inquiry, 13, 249-275.

Snyder, C. \& Lopez, S. (2002). A Handbook of Positive Psychology. New York: Oxford University Press.

Snyder, C. \& Lopez, S. (2007). Positive Psychology: The Scientific and Practical Explorations of Human Strengths. Thousand Oaks, California: Sage Publications.

Sternerg, R.J. \& Grigorenko, H.L. (2001). Unified psychology. American Psychologist, 56, 1069-1079.

Vaillant, G.E. (1977). Adaptation to lyfe. Boston: Little Brown. 
Vázquez, C. (2006). La Psicología Positiva en perspectiva. En Papeles del Psicólogo, 27, 1-2.

Vazquez, C. \& Hervás, G. (2008). Psicología Positiva Aplicada. Bilbao: Desclée de Brouwer

Vazquez, C. \& Hervás, G. (2009). La ciencia del bienestar. Fundamentos de una psicología positiva. Madrid: Alianza Editorial.
Vázquez, C., Sánchez, A. \& Hervás, C. (2008). Intervenciones psicológicas para la promoción del bienestar. En C. Vázquez y G. Hervás (Eds.), Psicología positiva aplicada (pp. 41-71). Bilbao: Desclee de Brower.

Watkins, P.C., Woodward, K., Stone, T., \& Kolts, R.L. (2003). Gratitude and happiness: development of a measure of gratitude, and relationships with subjective well-being. Social Behavior and Personality, 31(5), 431-452. 